\title{
Review of current trends in enhancing bioavailability of poorly water soluble drugs by liposomal interventions
}

\author{
Stephen Olaribigbe Majekodunmi \\ Department of Pharmaceutics and Pharmaceutical Technology,Faculty of Pharmacy, University of Uyo, P.M.B \\ 1017, Uyo, Nigeria
}

\begin{abstract}
One of the several ways of enhancing the solubility of poorly soluble drugs is liposomes formulations. Research on liposomes formulations has progressed from that of conventional vesicles to new generation liposomes, such as cationic liposomes, temperature sensitive liposomes, and virosomes, by modulating the formulation techniques and lipid composition. Thermosensitive liposomes are also a promising tool for external targeting of drugs to solid tumors when used in combination with local hyperthermia or high intensity focused ultrasound. In vivo results have demonstrated strong evidence that external targeting is superior over passive targeting achieved by highly stable long-circulating drug formulations like PEGylated liposomal doxorubicin. This review is to compare the therapeutic effect of current clinically approved liposomebased drugs with free drugs and summarize the historical development and effects of particular phospholipids and surfactants on the biophysical properties and in vivo efficacy of thermosensitive liposome formulations. Drug delivery guided by magnetic resonance imaging is also discussed. The combination of external targeting with thermosensitive liposomes and magnetic resonance-guided drug delivery will be the unique characteristics of this nanotechnology approach in medicine.
\end{abstract}

Keywords: Temperature sensitive liposomes, PEGylated liposomes, hyperthermia, drug targeting, virosomes

\section{INTRODUCTION}

Bioavailability, one of the principal pharmacokinetic properties of drugs, is used to describe the fraction of an administered dose of unchanged drug that reaches the systemic circulation. By definition, when a medication is administered intravenously, its bioavailability is $100 \%$. However, when a medication is administered via other routes (such as oral), its bioavailability decreases (due to incomplete absorption or firstpass metabolism). The measurement of the amount of the drug in the plasma at periodic time intervals indirectly indicates the rate and extent at which the active pharmaceutical ingredient is absorbed from the drug product and becomes available at the site of action. Bioavailability is expressed as either absolute or relative bioavailability [1].

Liposomes are vesicles formed by either synthetic or nature phospholipids. These phospholipid molecules arrange themselves spontaneously into bilayer structures in water, so that the hydrophobic tails are shielded from water by hydrophilic heads. Vesicles can consist of one or more phospholipid bilayers with hydrophilic and hydrophobic compartments. The hydrophobic compartments can carry or fill with hydrophobic or water-insoluble drugs while the hydrophilic compartments can load hydrophilic or water-soluble drugs. This is discussed much later in this review.

Apart from liposomes interventions, other methods used in enhancing solubility of poorly soluble compounds are complexation, cosolvents, emulsions, microemulsions, micelles, pharmaceutical salts, pro-drugs, particle size reduction, percolation, solid state alternation, soft gel technology and solid dispersion [2].

\subsection{Liposomes}

Liposomes are enclosed vesicles composed of phosphotidylcholine and cholesterol lipid bilayers, and have been extensively studied as drug carriers. Hydrophilic drugs can be loaded into the inner aqueous phase, whereas hydrophobic drugs can be inserted into the hydrophobic lipid bilayers. Although drug-loaded liposomes have been mainly used for the parenteral administrations, their potential application as oral drug delivery systems has also been studied. Oral liposomes may provide increased solubility of their load and protection from the hostile environment in the gastrointestinal tract [3], [4]. Most importantly, the similarity between liposomal lipid bilayers and biomembranes and the relatively small size of liposomes significantly facilitate oral absorption [5]. For example, oral administration of protein- or polypeptide-loaded liposomes has been shown to give rise to enhanced absorption of active biomacromolecules [3], [6], [7]. Incorporation of poorly permeable small molecule drugs into liposomes also yielded improved oral absorption [8], [9], [10]. In particular, for poorly water-soluble drugs, substantial enhancement in bioavailability or in vivo efficacy has been observed following liposomal encapsulation [11], [12], [13]. 
Liposomes are excellent candidates for drug delivery, because of their biocompatibility, the possibility to fine-tune their physico-chemical properties according to their lipid composition and content and, also, due to the possibility to tailor their surface composition [14]. These vesicles are indeed obtained from naturally occurring phospholipids or from synthetic mimetic lipids surrounding an aqueous core. Liposomes have initially been proposed by Gregoriadis [15], [16] and have been the only nanoparticles available on the market, since 2008. Their long-standing commercialization with various classes of molecules, such as doxorubicin, amphotericin B, verteporfin, cytarabine, vincristine or inactivated hepatitis B virus, give the liposomes an advantage over other nanocarriers, because of the technical and efficacy knowledge gathered over the years [16], [18]. Several companies have since been launched to optimize the processes and to produce good manufacturing process (GMP) liposome batches [19].

\subsection{Liposome containing bile salt}

The underlying mechanism for the facilitated oral absorption by liposomes has been partly elucidated by studies on the phase transition behavior of liposomal vesicles under simulated gastrointestinal environment, especially in the presence of bile salts. Physiological bile salts can interact with phospholipids in the gastrointestinal tract to form mixed micelles that play important roles in enhancing absorption of digestive lipids and poorly water-soluble drugs and alleviating membrane toxicity induced by bile salt monomers [20], [21], [22], [23]. Likewise, bile salt monomers can penetrate into liposomal lipid bilayers and disrupt the vesicular structure, and further increase in bile salt concentration can induce liposomes to undergo vesicle-micelle transition [21], [24]. The resultant mixed micelles have been shown to function as excellent vehicles for poorly water-soluble drug molecules and one of the most important mesophases before absorption [23]. Therefore, liposomes containing bile salts, also called transfersomes when originally developed for transdermal delivery [25], [26], [27], can readily transform into mixed micelles in the gastrointestinal environment, thus enhancing transmembrane absorption. Chen et al., [28] 2009, successfully enhanced the bioavailability of the poorly watersolube drug fenofibrate by using liposomes containing a bile salt, sodium deoxycholate, compared with the fast release formulation of micronized fenofibrate. In their work, the apparent disparity between between slow release of incorporated fenofibrate and quick peak time indicated mechanisms other than enhanced release by liposomes that contributed to improved bioavailability.

Although liposomes are considered to be able to entrap both hydrophilic and lipophilic drugs into their aqueous compartment and their lipidic bilayer, respectively [29], one can easily guess that lipophilic drugs will not be encapsulated with high efficiency without disturbing the membrane bilayer integrity [30].

Liposomes are the nanoparticles on which we have the most experience, due to products having already been commercialized. Due to this vast experience on liposomes, one would have expected more liposomal formulations to be put on the market, in correspondence to the vast literature on drug delivery with liposomes. Liposomes have tremendous advantages, like increased drug stability and bioavailability [28]. Furthermore, because of their size, liposomes can flow easily in blood circulation and sustain distortion, such as red blood cells. In addition, liposomes are retained in inflamed tissues, taking advantage of discontinuous endothelia. Besides their advantages, one of the main limitations of liposomes is their stability upon storage and rapid elimination after administration.

\subsection{Thermosensitive liposomal drug delivery systems}

Thermosensitive liposomes are a promising tool for external targeting of drugs to solid tumors when used in combination with local hyperthermia or high intensity focused ultrasound. In vivo results of the work of Kneidl et al., [31] have demonstrated strong evidence that external targeting is superior over passive targeting achieved by highly stable long-circulating drug formulations like PEGylated liposomal doxorubicin. Up to March 2014, the Web of Science listed 371 original papers in this field, with 45 in 2013 alone [31]. Several formulations have been developed since 1978, with lysolipid-containing, low temperature-sensitive liposomes currently under clinical investigation.

Yatvin et al., [32] described the first temperature-sensitive formulation (thermosensitive liposome, TSL) that was able to release a hydrophilic drug when the temperature was increased a few degrees above physiological temperature. The original formulation based on 1,2-dipalmitoyl-sn-glycero-3-phosphocholine (DPPC) and 1,2-distearoyl-sn-glycero-3-phosphocholine (DSPC) 3:1 (mol/mol) has been modified frequently during recent decades to overcome several limitations. At the beginning of the 21 st century, the first TSL formulation developed by Needham et al [33] entered human clinical trials [34]. This was a breakthrough in the field, visible by approximately 300 citations of the original paper [33] since its publication. Heat-triggered drug release from liposomes can also be achieved by adding thermosensitive polymers to the formulation [35]. However, in the present review, we focus on formulations where thermosensitivity is achieved by the biophysical properties of the membrane-forming phospholipids and highlight the influence of lipid composition on the in vitro and in vivo behaviour of the TSL formulations currently under investigation. This is in contrast 
with previously published reviews, which have concentrated on particular TSL formulations [33], [36], or image-guided drug delivery [37].

\subsection{Traditional temperature-sensitive liposomes}

Gaber et al., [38] reported the effect of cholesterol and PEG-phosphatidylethanolamine with regard to stabilizing TSL formulations in vitro. Incorporation of $30 \mathrm{~mol} \%$ cholesterol into TSL formulations eliminated $\mathrm{T}_{\mathrm{m}}$ by changing the phase state of the membrane to a liquid-ordered phase. Vesicles composed of DPPC/HSPC/cholesterol/DPPE-PEG 50:25:15:3 (mol/mol) released 60\% of their doxorubicin content during 30 minutes of incubation at $42^{\circ} \mathrm{C}$ in human plasma [38]. In vivo fluorescence video microscopy in rats revealed liposome extravasation [38]. The doxorubicin content in the interstitial space was negligible at $34^{\circ} \mathrm{C}$, but increased by 38 -fold when the tumor was heated to $42^{\circ} \mathrm{C}$ for one hour [38].. A traditional temperature-sensitive liposome (TTSL) formulation with coencapsulated doxorubicin and a gadolinium-based contrast agent for MRIguided delivery of doxorubicin is currently under investigation [39], [40], [41]. The TTSL formulation has been used in these studies because of its higher stability when compared with lysolipid-containing low temperaturesensitive liposome (LTSL) formulations [39].

\subsubsection{Lysolipid-containing low temperature-sensitive liposomes}

The breakthrough in development of clinically usable TSL formulations was the incorporation of lysolipids into the membrane bilayer [34]. The LTSL formulation was originally composed of DPPC/lysoPC/DSPE-PEG ${ }_{2000}$ 90:10:4 (mol/mol) but was modified slightly in recent years [33]. The surfactant, lyso-PC, mediates drug release around $\mathrm{T}_{\mathrm{m}}$ by formation of lysolipid-stabilized membrane pores [33], [42], [43]. The release rate of doxorubicin from LTSL at $41.3^{\circ} \mathrm{C}$ was $80 \%$ in 20 seconds. In comparison, TTSL released only $40 \%$ of its doxorubicin content in 30 minutes on heating to $42^{\circ} \mathrm{C}$ [34], [38].

Complete regression of tumors was achieved in a preclinical xenograft mouse model using doxorubicin encapsulated in LTSL [44]. In an orthotopic murine mammary model, reduction of blood flow and microvascular density occurred after local application of hyperthermia [45]. Four xenograft models have been studied, and show that LTSL had improved efficacy in comparison with TTSL [46]. Hauck et al., [47], published a Phase I study of LTSL performed in dogs with spontaneously grown solid tumors. The tumor response achieved supported further evaluation of this formulation, but the maximum tolerated dose of 0.93 $\mathrm{mg} / \mathrm{kg}$ was slightly lower than the published dose for free doxorubicin in dogs. The LTSL formulation with encapsulated doxorubicin (Thermodox ${ }^{\circledR}$ ) was licensed to Celsion Corporation (Columbia, MD, USA) and is currently under clinical investigation [33], [48]. Woo et al., [49] encapsulated cisplatin into LTSL, but until now only in vitro tests have been published. LTSL was the first TSL formulation suitable for use in the intravascular drug release approach. This formulation is characterized by ultrafast drug release upon heating, although incorporation of surfactants in the formulation decreases vesicle stability around $\mathrm{T}_{\mathrm{m}}$ [42]. Moreover, approximately $70 \%$ of lysolipid was found to dissociate from the formulation within one hour post injection [50].

\subsection{2 $\mathrm{DPPG}_{2}$-thermosensitive liposome}

In 2004, a new liposomal formulation (DPPG 2 -TSL) composed of the phospholipids DPPC, DSPC, and 1,2-dipalmitoyl-sn-glycero-3-phosphodiglycerol $\left(\mathrm{DPPG}_{2}\right)$, was reported by Lindner et al., [51]. $\mathrm{DPPG}_{2}$ is a synthetic phospholipid with a molecular weight close to that of natural occurring 1,2-dipalmitoyl-sn-glycero-3phosphoglycerol, because only one additional glycerol molecule is bound via an ether bond to the head group [51]. The molecular class of oligoglycerols $\mathrm{DPPG}_{\mathrm{n}}$ was developed to increase the circulation half-life of vesicles in the same way as for PEGylated lipids. Lasic et al [52] postulated that highly hydrated groups like PEG on the liposomal surface are capable of sterically inhibiting electrostatic and hydrophobic interactions with blood components. Incorporation of $\mathrm{DPPG}_{2}$ led to a prolonged circulation time in non-thermosensitive [53] and thermosensitive formulations [54], [55]. The plasma half-life of carboxyfluorescein encapsulated into $\mathrm{DPPG}_{2^{-}}$ TSL was reported to be 9.6 hours in hamsters and 5 hours in rats [51]. Because of the significantly smaller head group modification of the phospholipid compared to DSPE-PEG 2000 (74 Da versus approximately 2,000 Da), $\mathrm{DPPG}_{\mathrm{n}}$ forms lamellar structures and could be incorporated into TSL formulations with up to $70 \mathrm{~mol} \%$. Incorporation of DSPE-PEG 2000 instead is limited to concentrations below $10 \%$ mol, since it acts like a surfactant with a critical micelle concentration of $0.5-1.0 \mu \mathrm{M}$. The $\mathrm{T}_{\mathrm{m}}$ for $\mathrm{DPPG}_{2}-\mathrm{TSL}$ is around $42^{\circ} \mathrm{C}$, with a narrow transition range.

In contrast with the LTSL formulation, incorporation of surfactants into $\mathrm{DPPG}_{2}-\mathrm{TSL}$ was avoided. However, the release rates of carboxyfluorescein and doxorubicin from $\mathrm{DPPG}_{2}-\mathrm{TSL}$ were as fast as measured with the LTSL formulation [56], but drug release from the $\mathrm{DPPG}_{2}-\mathrm{TSL}$ formulation started at approximately one degree higher temperature [56], $\mathrm{DPPG}_{2}$-TSL showed improved in vitro stability in complete serum when compared with LTSL [57]. The presence of serum components at $37^{\circ} \mathrm{C}$ stabilized the formulation over time, 
whereas the opposite was found for LTSL. Interestingly, the lipid composition of a TSL formulation markedly influenced the effect of serum components on vesicle stability. DPPC/DSPC/DSPE-PEG 2000 80:15:5 (mol/mol) (Stealth TSL) and LTSL were more susceptible towards destabilizing effects by cholesterol-containing vesicles [57] whereas the presence of immunoglobulin type $G$ stronger affected the stability of DPPG 2 -TSL [57]. Moreover, the stability of $\mathrm{DPPG}_{2}$-TSL was less affected by size changes in the range of $100-150 \mathrm{~nm}$ compared to surfactant containing LTSL [58].

Incorporation of $10 \%$ mol hexadecylphosphocholine $(\mathrm{HePC})$ into the membrane of $\mathrm{DPPG}_{2}-\mathrm{TSL}_{\text {further }}$ increased the release rate of the encapsulated drug, similar to lyso-PC [55]. HePC is structurally related to lysoPC, but has better chemical and metabolic stability, and is approved as a lipophilic drug for the treatment of skin metastasis in breast cancer and for leishmaniasis. The in vitro cytotoxicity of HePC in DPPG $_{2}$-TSL was heatinducible and stronger than that induced by micellar HePC, which did not respond to heat [55].

Limmer et al., [54] passively loaded gemcitabine into DPPG $_{2}-\mathrm{TSL}$, and their pharmacokinetic studies in rats using gemcitabine $6 \mathrm{mg} / \mathrm{kg}$ body weight showed an initial plasma half-life of 0.53 hours for gemcitabine encapsulated in $\mathrm{DPPG}_{2}-\mathrm{TSL}$, with a size of $109 \mathrm{~nm}$. The plasma half-life was increased to 2.59 hours when the vesicle size was increased to $129 \mathrm{~nm}$. In a therapeutic study, significant delay of tumor growth was found for heat-triggered gemcitabine from $\mathrm{DPPG}_{2}-\mathrm{TSL}$ when compared with other gemcitabine formulations, including gemcitabine encapsulated in $\mathrm{DPPG}_{2}$-TSL without hyperthermia [54].

$\mathrm{DPPG}_{2}$-TSL is currently the only TSL formulation that fulfills all the criteria for heat-triggered intravascular drug release. Drug release upon activation from this formulation is comparable fast as observed with LTSL formulation. Moreover, the absence of surfactants yields a long-circulating formulation, with high plasma levels after intraveneous application for the duration of a typical hyperthermia treatment in the clinic.

\subsubsection{Stealth TSL}

A sterically stabilized TSL formulation (Stealth TSL) was developed from the original Yatvin formulation by adding DSPE-PEG 2000 for improved stability and a better in vivo half-life when compared with the LTSL formulation [56], and enabled passive accumulation of TSL in tumor tissue [36] and compared Stealth TSL and LTSL, and found that the former had superior in vitro stability at $37^{\circ} \mathrm{C}$ in serum [36]. The maximum release of doxorubicin from Stealth TSL was at $42^{\circ} \mathrm{C}$ [36]. In comparison with LTSL, release of doxorubicin from Stealth TSL starts at higher temperatures $\left(39^{\circ} \mathrm{C}\right.$ versus $\left.37^{\circ} \mathrm{C}\right)$ [36]. Because of the absence of lyso-PC in Stealth TSL, the rate of release of doxorubicin at $42^{\circ} \mathrm{C}$ was slower $(75 \%$, one minute) when compared with LTSL (99\%, one minute) [36]. In a BFS-1 mouse model, Stealth TSL showed improved tumor growth control over LTSL when combined with mild hyperthermia [36].

\subsubsection{Hyperthermia-activated cytotoxic formulation}

Another TSL formulation with encapsulated doxorubicin currently under investigation is the hyperthermia-activated cytotoxic (HaT) liposome formulation described by Tagami et al., [59]. HaT is composed of DPPC and the nonionic surfactant, polyoxyethylene (20) stearyl ether (Brij78) Brij78 consists of a PEGylated acyl chain, so it was hypothesized that Brij78 could replace the function of lyso-PC and DSPE$\mathrm{PEG}_{2000}$ in the LTSL formulation [59]. The HaT formulation showed 100\% doxorubicin release within 3 minutes at a temperature of $40^{\circ} \mathrm{C}-42^{\circ} \mathrm{C}$ in buffer [59]. In comparison with LTSL, HaT showed enhanced drug release rates at $40^{\circ} \mathrm{C}$, with similar blood pharmacokinetics [59]. For both formulations, a blood circulation halflife of approximately 0.5 hours was observed after injection [59]. A single intravenous treatment with HaT at a doxorubicin dose of $3 \mathrm{~m}) \mathrm{g} / \mathrm{kg}$ body weight in combination with local hyperthermia showed enhanced tumor regression when compared with LTSL [59].

Gemcitabine and oxaliplatin have also been encapsulated into the HaT formulation. In a pharmacokinetic study in mice, $40 \%$ of the injected dose was detectable 2 hours after intravenous administration of gemcitabine encapsulated in HaT [60]. For oxaliplatin, a three-fold reduction in clearance was observed in comparison with the free drug. HaT showed a 25 -fold improvement in delivery of gemcitabine to the heated tumor relative to free gemcitabine [60]. Unfortunately, superiority of external targeting was not shown in the therapeutic study, because there was no appropriate control group (i.e. HaT without hyperthermia).

Tagami et al., [61] reported an improved method for active loading of doxorubicin into the HaT formulation based on a copper (II) gradient (HaT-II). HaT-II showed improved in vitro stability at $37^{\circ} \mathrm{C}$, together with a faster drug release rate at $41^{\circ} \mathrm{C}$ in the presence of serum when compared with LTSL. In comparison with LTSL, HaT-II showed a 2.5-fold longer blood circulation time in mice and a 2.0 -fold increase in drug delivery to the heated tumor. This resulted in improved antitumor efficacy [61]. 


\subsubsection{STL formulation}

Park et al., [62] reported another stabilized formulation composed of DPPC, DSPE-PEG ${ }_{2000}$, cholesterol, and fatty acid-conjugated elastin-like polypeptide 55:2:15:0.4125 (mol/mol) (STL) with encapsulated doxorubicin. Pharmacokinetic studies in mice showed plasma half-lives of 2.03 hours and 0.92 hours for doxorubicin encapsulated in STL and LTSL respectively. In combination with high intensity focused ultrasound, STL achieved significantly better tumor growth delay 7 days after injection when compared with LTSL [62].

\subsubsection{Thermosensitive liposomes for mri-guided drug delivery}

MRI is the method of choice for image-guided drug delivery with TSL. Its abilities with regard to morphological and functional tumor characterization without exposure to ionizing radiation are well known, and it is a standard method in clinical use. Further, MRI thermometry is established for the control of thermotherapies, such as radiofrequency hyperthermia and high intensity focused ultrasound. Dedicated hybrid systems have already been introduced into clinical applications [63], [64]. Localized drug release from TSL has been demonstrated in rodents [40], [65] and nonrodents [66], [67] using MRI for the control of hyperthermia. Beyond controlling the volume of heating, encapsulation of MRI contrast agents in TSL formulations allows additional characterization of the drug delivery only accessible in humans when using MRI.

\subsubsection{Signal mechanism}

Paramagnetic gadolinium chelates are typical MRI-active contrast agents for encapsulation in TSL formulations [39], [68], [69], [70], [71], [72], [73], [74], [75]. The nuclear magnetic resonance of water protons is the primary origin of MRI signal and not the contrast agent itself. MRI contrast agents are only visualized by their ability to accelerate the water proton relaxation in the vicinity of the contrast agent molecules. This indirect signal forming process is only effective if the contrast agent molecule is allowed to interact with a large number of water protons. For visualization of temperature-induced release, the contrast agent has to be encapsulated inside the TSL. [76], [77]. Below the $\mathrm{T}_{\mathrm{m}}$, the contrast agent interacts mainly with the water present inside the TSL, because water exchange with the exterior of the TSL is limited. As a result, the visibility of the contrast agent is reduced when compared with free contrast agents. When approaching the $T_{m}$, the increase in water exchange results in a signal increase in $T_{1}$-weighted images [69]. Around the $T_{m}$, the contrast agent is released and the signal change is maximal and comparable with the signal change achieved with free contrast agent [69], [71], [76], [77]. This makes it possible to strongly change an MRI signal by altering temperature [77].

The maximum achievable signal change depends on the type of contrast agent [71], lipid composition [71], [76], vesicle size [76] and concentration of the encapsulated contrast agent [71], [76]. The heating method might also play a role, e.g. focused ultrasound adds a mechanical release component to the signal mechanism [37]. The signal mechanism described in the paragraph before is mainly related to the contrast agent induced change of the longitudinal $\left(\mathrm{T}_{1}\right)$ relaxation time of the water protons. But signal formation in MRI is complex, often showing a weighted signal depending not only on the $\mathrm{T}_{1}$ relaxation time shortening effect but also on the type of pulse sequence, choice of sequence parameters, and effects related to $T_{2}$ relaxation. Quantification strategies in MRI thus try to determine a single parameter, such as the $T_{1}$ relaxation time, with the aim of being independent of variables such as system settings of an individual measurement or in homogeneity of the receiver coil. $T_{1}$ relaxation remains the parameter of choice because it is directly related to the membrane dynamics.

\section{APPLICATIONS OF LIPOSOMES}

TSL can be applied with an encapsulated contrast agent to distinguish heated from unheated tissue [70], [78], or to quantify absolute temperatures complementing traditional MRI thermometry methods [79], [80] thus serving as a tool for quality assurance in thermotherapy in patients.

It has been demonstrated that potentially quantitative estimation of drug release based on $\mathrm{T}_{1}$ relaxation time changes is possible if the contrast agent and drug are both encapsulated in a TSL formulation [40], [72], [73], [74], [59] thus allowing "drug dose painting" [74], or "chemodosimetry" [73]. Because the drug itself is not observed by MRI, a correlation between the contrast agent and drug release had to be established. For that purpose, LTSL were actively loaded with doxorubicin using a manganese (II) gradient [72]. Doxorubicin and manganese (II) form a stable complex [81] with the paramagnetic manganese (II) serving as an MRI contrast agent. Thus the release kinetics are the same for the contrast agent and the drug, allowing for correlation between change in $\mathrm{T}_{1}$ relaxation time (determined by MRI) and amount of doxorubicin (determined by highperformance liquid chromatography). Using this strategy, it was possible to show that release of doxorubicin was heterogeneously distributed in the tumor model, and that LTSL administered during hyperthermia had the greatest antitumor effect when compared with other administration strategies. The major drawback of the above approach is the toxicity related to manganese(II). 
To overcome this, other researchers are using clinically approved gadolinium-based contrast agents.

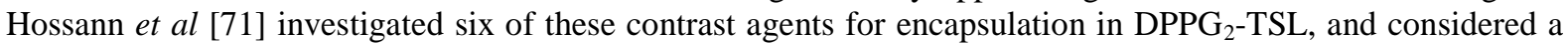
nonionic contrast agent with a low contribution to osmolality to be optimal. Two strategies of encapsulation are possible using gadolinium-based contrast agents, but the release kinetics and signal mechanisms for both the contrast agent and drug have to be considered. One strategy is to combine two subsets of TSL, with one encapsulating only the contrast agent and a second encapsulating only the drug [69]. This strategy allows a higher amount of contrast agent and drug to be encapsulated whilst avoiding osmotic effects [71]. The second strategy is to coencapsulate both drug and contrast agent in the same TSL [39], [40], [41], [68], [67], [59], which limits the amount of both components in each TSL. Nevertheless, for both strategies, it has to be ensured that the temperature-dependent drug release rate and MRI signal change are correlated [68].

An important risk associated with clinical application of a gadolinium-based contrast agent is nephrogenic systemic fibrosis, a rare side effect in patients. The pathophysiological mechanism involves a reduced glomerular filtration rate and a long retention time combined with transmetallation of gadolinium (III). Hijnen et al [65] addressed this concern in a rat model, but could not detect dissociation of gadolinium-DTPA in high intensity focused ultrasound ablation therapy. De Smet et al [41] investigated the blood kinetics and biodistribution of in-labeled TTSL with coencapsulated doxorubicin and gadolinium- $\left(\mathrm{HPDO}_{3} \mathrm{~A}\right)\left(\mathrm{H}_{2} \mathrm{O}\right)$ in Fisher rats and found significant clearance with $\leq 0.3 \%$ of the injected dose in all analyzed organs one month after injection. Nevertheless, before application in humans, further investigation of the risks associated with this strategy seems necessary.

\section{STORAGE OF LIPOSOMES}

Liposomes dispersed in aqueous solution generally face physical and chemical instabilities after longterm storage [82]. Hydrolysis and oxidation of phospholipids and liposome aggregation are the common cause of liposome instabilities. According to the literature, many methods have been investigated for the stabilization of liposomes, such as lyophilization, freezing, and spraying drying. In commercial liposome-based drugs, AmBisome ${ }^{\circledR}$ (Gilead Sciences, Inc, San Dimas, CA), Amphotec ${ }^{\circledR}$ (Ben Venue Laboratories, Inc, Bedford, OH), Myocet, Visudyne ${ }^{\circledR}$ (Novartis Pharma AG, Basel, Switzerland), and LEP-ETU (liposome-entrapped paclitaxel easy-to-use; NeoPharm, Inc, Lake Bluff, IL) are all lyophilized products. In general, freeze-drying increases the shelf-life of liposomal formulations and preserves them in dried form as lyophilized cakes to be reconstituted with water for injection prior to administration [83]. Furthermore, cryoprotectants need to be added to maintain particle size distribution of liposomes after the freeze-drying-rehydration cycle. Various types and concentrations of sugars have been investigated for their ability to protect liposomes against fusion and leakage during lyophilization processes [83]. In commercial liposome lyophilized products, lactose has been used as a cryoprotectant in the formulations of Amphotec, Myocet, and Visudyne, and sucrose was added in the formulations of AmBisome and LEP-ETU to increase liposome stability during lyophilization.

Clemons and Stevens (1998) compared the potency and therapeutic efficacy among the different lipidbased formulations of amphotericin B (Amphotec, AmBisome, and Abelcet ${ }^{\circledR}$ (Sigma-Tau PharmaSource, Inc, Indianapolis, IN)) for the treatment of systemic and meningeal cryptococcal disease. Their work indicated that the therapeutic efficacy of Amphotec and AmBisome was superior to that of Abelcet, by up to ten-fold, in survival and in clearing infection from all organs. In these three commercially available lipid-based formulations of amphotericin B, Amphotec and AmBisome are both lyophilized products and Abelcet is formulated as a suspension. Therefore, lyophilization may not extend the shelf-life of products but may increase therapeutic efficacy in vivo. Similar results were also reported in our previous studies [84]. The stability of the siRNAloaded liposomes in suspension and lyophilized powder form up to 1 month postmanufacture was investigated [85]. Following formulation, the siRNA-loaded liposomes were stored at either $4{ }^{\circ} \mathrm{C}$ or room temperature. The particle size and zeta potential of siRNA-loaded liposomes remained unchanged in both storage conditions. However, siRNA entrapment efficiencies were observed to have decreased slightly after 1 month in storage for both suspension $(90 \% \rightarrow 83 \%)$ and lyophilized powder $(94 \% \rightarrow 84 \%)$ forms. Surprisingly, the gene-silencing efficiency of siRNA-loaded liposomes in aqueous solution showed $80 \%$ reduction following 1 month of storage at either $4^{\circ} \mathrm{C}$ or room temperature. This was in contrast to liposomes prepared in the lyophilized powder form where $100 \%$ of the gene-silencing efficiency was retained following storage at either $4^{\circ} \mathrm{C}$ or room temperature for 1 month. Although therapeutic efficiency of liposome-based drugs may vary depending on the choice of lipids, the preparation technique, physico-chemical characteristics of the bioactive materials, and overall charge of the liposome, lyophilization is useful for the long-term storage of liposome-based drugs.

\section{CONCLUDING REMARKS}

Poor bioavailability is a major limitation in successful drug delivery by oral route. Lot of research work is focused on oral and parenteral bioavailability enhancement of the poorly absorbed drugs by liposomes. Since 
the first liposomal pharmaceutical product, Doxil, received FDA approval in 1995, liposomes have been widely used as drug carriers in clinic. Until now, several important types of liposomes, such as PEGylated liposomes (Doxil and Lipo-dox), temperature sensitive liposomes (ThermoDox), cationic liposomes (EndoTAG1-1), and virosomes (Expal and Inflexal V) have been investigated for clinic use. New liposomal formulations, such as PEGylated liposomes, may extend blood circulation time, vary drug distribution in the body, and hence reduce the possible side effects related to the drugs (eg, cardiotoxicity). However, PEGylated liposomes (Doxil and Lipo-dox) displayed significant incidence of stomatitis in clinical trials, which may be related to PEGylation. Moreover, some of the new generation liposomes showed only comparable or even poor therapeutic efficiency compared with free drug or conventional vesicles in clinical trials. In comparison with Doxil, ThermoDox displayed significantly weaker doxorubicin accumulation in mice tumors at 24 hours after administration. EndoTAG-1 plus gemcitabine and EndoTAG-1 plus paclitaxel achieved excellent therapeutic effect in two Phase II clinical trials in pancreatic cancer and triple receptor-negative breast cancer, but EndoTAG-1 therapy alone in triple receptor-negative breast cancer resulted in poor survival rate (34\%) and median progression-free survival time (3.4 months) in comparison with paclitaxel (48\% and 3.7 months). SPI-077, the first liposomal formulation of cisplatin, had limited clinical efficacy in a Phase II clinical trial of advanced non-small cell lung cancer, even though SPI-077 demonstrated enhanced cisplatin tumor accumulation in preclinical models. Temperature sensitive liposomes (TSL) are a promising tool for external targeting of drugs to solid tumors in combination with local hyperthermia or high intensity focused ultrasound. Several formulations have been developed, with one currently under investigation. In vivo results show strong evidence that external targeting is superior over passive targeting of highly stable long-circulating drug formulations. Moreover, MRI-guided drug delivery adds the possibilities.

\section{REFERENCES}

[1] L. Shargel, A. B. Yu, Applied biopharmaceutics \& pharmacokinetics (4th edition) New York: McGraw-Hill. ISBN 08385-0278-4, 1999.

[2] S. I. F. Badawy, M. M. Ghorab, C. M. Adeyeye, Characterization and bioavailability of danazolhydroxypropyl- $\beta$ cyclodextrin coprecipitates. Int. J. Pharm., Vol. 128, No. 1- 2, 1999, 45-54.

[3] A. Arien, C. Goigoux, C. Baquey, B. Dupuy, Study of in vitro and in vivo stability of liposomes loaded with calcitonin or indium in the gastrointestinal tract. Life Sci. 53, 1279-1290, 1993.

[4] A. Arien, N. Henry-Toulmé, B. Dupuy, Calcitonin-loaded liposomes: stability under acidic conditions and bile salts-induced disruption resulting in calcitonin-phospholipid complex formation. Biochim. Biophys. Acta 1193, 1994, 93-100.

[5] B. J. Aungust, Novel formulation strategies for improving oral bioavailability of drugs with poor membrane permeation or presystemic metabolism. J. Pharm. Sci. 82, 1993, 979-987.

[6] G, Dapergolas, G. Gregoriadis, Hypoglycaemic effect of liposome entrapped insulin administered intragastrically into rats. Lancet 2, 1976, 824-827.

[7] Z. Degim, N. Unal, D. Essiz, U, Abbasoglu, The effect of various liposome formulations on insulin penetration across Caco-2 cell monolayer. Life Sci. 75, 2004, 2819-2827.

[8] W. Sun, W. Zou, G. Huang, A. Li, N. Zhang, Pharmacokinetics and targeting property of TFu-loaded liposomes with different sizes after intraveneous and oral administration. J Drug Target. 16, 2008, 357-365.

[9] V. Moutardier, F. Tosini, P. Vlieghe, L. Cara, J. R. Delpero, T. Clerc, Colloidal anticancer drugs bioavailability in oral administration models. Int J Pharm. 260, 2003, 23-38.

[10] . D. Deshmukh, W. R. Ravis, G. V. Betageri, Improved delivery of cromolyn from oral proliposomal beads. Int J Pharm, 358, 2008, 128-136.

[11] S. C. Mourao, P. I. Costa, H. R. Salgado, M. P. Gremiao, Improvement of antischistosomal activity of praziquantel by incorporation into phosphatidylcholine containing liposomes. Int. J. Pharm 295, 2005, 157162.

[12] J. Guo, Q. Ping, Y. Chen, Pharmacokinetics of cyclosporine A in rabbits by oral administration of lecithin vesicle and sandimmun neural. Int. J. Pharm. 216, 2001, 17-21.

[13] Y. Y. Xiao, Y. M. Song, Z. P. Chen, Q. N. Ping, Preparation of silymarin proliposome: a new way to increase oral bioavailability of silymarin in beagle dogs. Int. J. Pharm. 319, 2006, 162-168.

[14] N. Mignet, J. Seguin, G. G. Chabot, Bioavailability of Polyphenol Liposomes: A Challenge Ahead. Pharmaceutics 5(3), 2013, $457-471$.

[15] K. Yamashia, T. Nakate, K. Okimoto, A. Ohike, Y. Tokunnaga, R. Ibuki, K. Higaki, T. Kimura, Establishment of new preparation method for solid dispersion formulation of tacrolimus. Int. J. Pharm. 267, 2003, 79-91.

[16] T. Higuchi, M. Ikeda, Rapidly dissolving forms of digoxin: hydroquinone complex, J. Pharm. Sci. 63(5), 1974, 809-811.

[17] E. Nelson, E. L. Knoechel, W. E. Hamlin, J. G. Wagner, Influence of the absorption rate of tolbutamide on the rate of decline of blood sugar levels in normal humans. Int J Pharm. 51, 1962, 509-514.

[18] S. L Lin, L. Lachman, C. J. Swartz, C. F. Heubner, Preformulation investigation 1. Relation of salt forms and biological activity of an experimental antihypertensive. J. Pharm.Sci., $\quad 61(9), 1972,1418-1422$. 
[19] T.Vasconcelos, B. Sarmento, P. Costa, 'Solid dispersion as strategy to improve oral bioavailability of poor water soluble drugs, ’Drug Discov Today, Vol 12 issues 23 - 24, 2007, 1068 - 1075.

[20] E. J. Dial, S. H. Rooijakkers, Darling, RL; Romero, JJ; Lichtenberger, LM. Role of phosphatidylcholine saturatoion in preventing bile salt toxicity to gastrointestinal epithelia and membrane. J Enterogastrol Hepatol, 23, 2008, 430436.

[21] A. Hildebrand, K. Beyer, R. Neubert, P. Garidel, A. Blume, Temperature dependent interaction of cholate and deoxcholate with fluid model membranes and their solubilization into mixed micelles. Colloids Surf: Biointerfaces, 32, 2003, 335-351.

[22] Martin, GP and Marriott, C. Membrane damage by bile salt: the protective function of phospholipids. J. Pharm. Pharmacol. 33, 1981, 754-759.

[23] C. J. Porter, N. L. Trevaskis, W. N. Charman, Lipid and lipidbased formulations: optimizing the oral delivery of lipophylic drugs. Nat Rev Drug Discov. 6, 2007, 231-248.

[24] S. Andrieux, L. Forte, S. Lesieur, M. Paternostre, M. Ollivon, Gabrielle-Madelmont, C. Solubilization of dipalmitoylphosphatidylcholine bilayers by sodium taurocholate: a model to study the stability of liposomes in the gastrointestinal tract and their mechanism of interaction with a model bile salt. Eur. J. Pharm. Biopharm, 71, 2009, 346-355.

[25] G. Cevc, Lipid vesicles and other colloids as drug carriers on the skin. Adv. Drug Deliv. Rev.56, 004, 675-711.

[26] G. Cevc, D. Gebauer, J. Stieber, A. Schätzlein, G. Blume, Ultraflexible vesicles, transfersomes, have an extremely low pore penetration resistance and transport therapeutic amounts of insulin across the intact mammalian skin. Biochim. Biophys. Acta, $\quad 1368,1998,201-215$.

[27] G. Cevc, U. Vierl, S. Mazgareanu, Functional characterisation of novel analgesic product based on selfregulating drug carriers. Int. J. Pharm. 360, 2008, 18-28.

[28] N. Maurer, D. B. Fenske, P. R. Cullis, Developments in liposomal drug delivery systems. Expert Opin Biol Ther. 1, 2001, 923-947.

[29] [29] A. Fahr, P. Van Hoogevest, S. May, N. Bergstrand, M. L. Leigh, Transfer of lipophylic drugs between liposomal membranes and biological interfaces: consequences for drug delivery. Eur J Pharm Sci. 26(3-4), 2005, 251-265.

[30] Y. Chen, Y. Lu, J. Chen, J. Lai, J. Sun, F. Hu, W.Wu, Enhanced bioavailability of the poorly water-soluble drug fenofibrate by using liposomes containing a bile salt. Int J Pharm. 376, 2009, 153-160.

[31] B. Kneidl, M. Peller, G. Winter, H. Lindner, M. Hossann, Thermosensitive liposomal drug delivery system: state of the art review. Dovepress, Vol 9(1), 2014, 4387-4398.

[32] M. B. Yatvin, J. N. Weinstein, W. H. Dennis, R. Blumenthal, Design of liposomes for enhanced local release of drugs by hyperthermia, Science, 202, 1978, 1290-1293.

[33] C. D. Landon, J. Y. Park, D. Needham, M. W. Dewhirst.. Nanoscale drug delivery and hyperthermia: the materials design and preclinical and clinical testing of low temperature-sensitive liposomes used in combination with mild hyperthermia in the treatment of local cancer, Open Nanoed J., 3, 2011, 38-64.

[34] D. Needham, G. Anyarambhatia, G. Kong, M. W. Dewhirst, A new temperature - sensitive liposomes for use with mild hyperthermia: characterization and testing in a human tumor xenograft model, Cancer Res., 60, 2000, $1197-1201$.

[35] M. Van Elk, R. Deckers, C. Oerlemans, et al. Triggered release of doxorubicin from temperature-sensitivepoly(N-(2hydroxypropyl)-methacrylamidemono/dilactate)graftedliposomes. Biomicromolecules. 15, 2014, 1002-1009.

[36] L. Li, T. L. Hagen, M. Bolkestein et al. Improved intratumoral nanoparticle extravasation and penetration by mild hyperthermia. J. Control Release, 167, 2013, 130-137.

[37] H. Grull, and S. Langereis, Hyperthermia-triggered drug delivery from temperature - sensitive liposomes using MRI- guided high intensity focused ultrasound, J. Control Release, 161, 2012, 317 - 327.

[38] M. H. Gaber, K. Hong, S. K. Huang, D. Papahadjopoulos. Thermosensitive sterically sterilized liposomes: formulation and in vitro studies on doxorubicin release by bovine serum and human plasma, Pharm. Res., 12, 1995, $1407-1416$

[39] M. De Smet, V. Langereis, H. Grull, Temperature - sensitive liposomes for doxorubicin delivery under MRI guidance, J. Control Release, 143, 2010, 120-127.

[40] M. De Smet, E. Heijman, S. Langereis, N. M. Hijnen, H. Grull, Magnetic resonance imaging of high intensity focused ultrasound mediated drug delivery form temperature - sensitive liposomes: an in vivo proof -of -concept study, J. Control Release, 150, 2011, 102-110.

[41] M. De Smet, S. Langereis, S. Van den Bosch, et al. SPEC/CT imaging of temperature-sensitive liposomes for MRimage guided drug delivery with high intensity focused ultrasound, J. Control Release, 169, 2013, 82-90.

[42] L. M. Ickenstein, M. C. Arfvidsson, D. Needham, L. D. Mayer, K. Edwarda, Disc formationin olesterol- free liposomes during phase transition, Biochim Biophys Acta, 1614, 2003, 135-138.

[43] J. K. Mills and D. Needham, Lysolipid incorporation in dipalmitoylphosphatidylcholine bilayer membrane enhances the ion permeability the drug release rates at the membrane ratetransition. Biochim Biophys Act. 1716, 77 - 96.

[44] D. Needham, M. W. Dewhirst, The development and testing of a new temperature- sensitive drug delivery system for the treatment of solid tumours, Adv Drug Deliv Rev., $\quad 53,2001,285$ - 305.

[45] Q. Chen, A. Krol, A. Wright, D. Needham, M. W. Dewhirst, F. Yuan, Tumor microvascular permeability is a key determinant for antivascular effects of doxorubicine encapsulated in a temperature-sensitive liposome. Int. J Hyperthermia, 24, 2008, $475-482$.

[46] P. S. Yarmolenko, Y. L. Zhao, C. Landon et al. Comparative effects of thermosensitive doxorubicin-containing liposomes and hyperthermia in human and murine tumours. Int J Hyperthermia, 26, 485 - 498, 2010. 
[47] M. L. Hauck, S. M. LaRue, W. P. Petros et al. Phase 1 trial of doxorubicin - containing low temperature sensitive liposomes in spontaneous canine tumours. Clin Cancer Res. 12, 2006, $4004-4010$

[48] B. J. Wood, R. T. Poon, J. K. Lockloin et al. Phase 1 study of heat-deployed liposomal doxorubicin during radiofrequency ablation for hepatic malignances. J Vasc Interv Radiol, 23, 2012, 248 - 255.

[49] J. Woo, G. N. Chu, G. Karisson et al. Use of a passive equilibration methodology to encapsulate cisplatin into preformed thermosensitive liposomes. Int J Pharm. 349, 2008, 38 - 46.

[50] B. Banno, L. M. Ickenstein, G. N. Chiu et al. the functional role of polyethylene glycol- lipid and lysolipid in the drug retension and release from lysolipid-containing thermsensitive liposomes in vitro and in vivo, J Pharm Sci. 99, 2010, $2295-2308$.

[51] L. H. Lindner, M. E. Eichhorn, H. Eibl et al. Novel temperature-sensitive liposomes with prolonged circulation time. Clin Cancer Res., 10, 2004, 2168 - 2178.

[52] D. D. Lasic, F. J. Martin, A. Gabizon, S. K. Huang, D. Papahadjopoulos, Sterically stabilized liposomes: a hypothesis on the molecular origin of the extended circulation times. BiochimBiophyscta. 1070, 1991, $187-192$.

[53] O. Schagon, Liposomen als potentielle Arzneistofftrager: Variation der biopharmzeutischen Eigenschaften durch 1,2-Dipalmitoyi-sn-glycero-oligo-glycerine. [Liposomes as drug carriers-variation of biopharmaceutical characteristics by incorporation of 1,2-dipalmiyoyl-sn-glycero-3-phospho-oligoglycerols]. Aachen,

Germany: Shaker Verlag; 1997, German.

[54] S. Limmer, J. Hahn, R. Schmidt et al. Gemcitabine treatment of rat soft tissue sarcoma with phosphotidyldiglycerol-based thermosensitive liposomes, Pharm Res, Epub 2014.

[55] L. H. Lindner, M. Hossann, M. Vogeser. et al. Dual role of hexadecylphosphocholine (miltefosine) in thermosensitive-liposomes: active ingredient and mediator of drug release, J Control Release, 125, 2008, 112 120.

[56] M. Hossann, M. Wiggenhorn, A. Schwerdt et al. In vitro stability and content release properties of phosphatidylglycerol containing thermosensitive liposomes. Biochim Biophys Acta, 1768, 2007, 2491 - 2499.

[57] M. Hossann, Z. Syunyaeva, R. Schmidt, et al. Proteins and choleatrol lipid vesicles are mediators of drug release from thermosensitive liposomes, J. Control Release, 162, 2012, 400-406, 2012.

[58] M. Hossann, T. Wang, M. Wiggenhorn et al. Size of thermosensitive liposomes influences content release, J. Control Release. 147, 2010, 436 - 443, 2010.

[59] T. Tagami, W. D. Foltz, M. J. Ernsting et al. MRI monitoring of intratumoral drug delivery and prediction of the therapeutic effect with a multifunctional thermosensitive liposomes. Biomaterials 32, 2011, $6570-$ 6578.

[60] J. P. May, M./ J. Ernsting, E. Undzys, S. D. Li, Thermosensitive liposomes for the delivery of gemcoitabine and oxaliplatin to tumors, Mol Pharm. 10, 2013, 4499-4508.

[61] T. Tagami, J. P. May, M. J. Ernsting, S. D. Li, A thermosensitive liposomes prepared with a Cu2+ gradient demonstrate improved pharmacokinetics, drug delivery and antitumour efficacy. J Control Release. 161, 2012, 142 $-149$.

[62] S. M. Park, M. S. Kim, S. J. Park et al., Novel temperature-triggered liposomes with high stabilityformulation, in vitro evaluation, and in vivo study combined with high intensity focused ultrasound (HIFU), J Control Release. 170, 2013, 373 - 370

[63] M. Peller, R. Loffler, A. Baur et al. [MRI-controlled regional hyperthermia]. Radiologe. $\quad 39, \quad 1999, \quad 756-\quad 763$, Grerman.

[64] Gellermann, J; Wlodarczyk, W; Hilderbrandt, B. et al. Noninvasive magnetic resonance themography of recurrent rectal carcinoma in a 1.5 Tesla hybrid system. Cancer Res. $\quad 65,2005,5872-5880$.

[65] N. M. Hijnen, E. Heijman, M. O. Kohler et al. Tumor hyperthermia and ablation in rats using a clinical MRHIFU system equipped with a dedicated small animal set up. Int J Hyperthermia. 28, 2012, 1412 - 155.

[66] R. Staruch, R. Chopra, K. Hynynen, Localised drug release using MRI-controlled focused ultrasound hyperthermia. Int J Hyperthermia. 27, 2011, 156-171.

[67] A. Ranjan, G. C. Jacobs, D. L. Woods et al. Image-guided drug delivery with magnetic resonance-guided high intensity focused ultrasound and temperature sensitive liposomes in a rabbit Vx2 tumor model. J Control Release. 158, 2012, $487-494$.

[68] A. H. Negussie, P. S. Yarmolenko, A. Partanen et al. Formulation and characterization of magnetic resonance imageable thermerlly sensitive liposomes for use with magnetic resonance - guide high intensity focused ultrasound. Int J Hyperthermia. 27, 2011, 140- 155.

[69] T. T. Wang, M. Hossann, H. M. Reinl et al. In vitro characterization of phosphatidylglyceroglycerol-based thermosensitive liposomes with encapsulated H-1 MR $\quad$ T-1 shortening gadodiamide. Contrast Media Mol Imaging. 158, 2008, $487-494$.

[70] M. Peller, A. Schwerdt, M. Hossann et al. MR-charactetization of mild hyperthermia- induced gadodiamine release from thermosensitive liposomes in solid tumor. Invest Radiol. 43, 2008, 877 - 892.

[71] M. Hossann, T. Wang, Z. Syunyaeva et al. Non-ionic Gd-based MRI contrast agents are optimal for encapsulation into phosphatidyldiglycerol-based thermosensitive liposomes. J Control Release. 166, 2013, 22 -29 .

[72] B. L. Viglianti, S. A. Abraham, C. R. Michelich, et al. In vivo monitoring of tissue pharmacokinetics of liposome/drug using MRI: illustration of targeted delivery. Magn $\quad$ Reson Med. 51: 2004, 1153 - 1162.

[73] B. L. Viglianti, A. M. Ponce, C. R. Michelich, et al. Chemodosimetry of in vivo tumor liposomal drug concentration using MRI. Magn Reson Med. 56, 2006, 1011 - 1018. 
[74] A. M. Ponce, B. L. Viglianti, D. Yu et al. Magnetic resonance imaging of temperature- sensitive liposomes release: drug dose painting and antitumor effects. J Natl Cancer Inst. 99, 2007, $53-63$.

[75] H. M. Reinl, M. Hossann, L. H. Lindner, LH; Reiser, M. Thermosensitive Mn2+ liposomes for MR-guided hyperthermia-solvent-dependent $\mathrm{Mn} 2+\mathrm{n}$ release. IFMBE Proc. $25,2010,21-24$.

[76] S. L. Fossheim, A. K. Fahlvik, J. Klaveness, F. N. Muller, Paramagnetic liposomes as MRI contrast agents: influence of liposomal physicochemical properties on the in vitro relaxivity.MagnResonImaging. 17, 1999, 83 -89 .

[77] S. L. Fossheim, K. A. Ilyasov, J. Hennig, A. Bjornerud, Thermosensitive paramagneticliposomes for temperature control during MR imaging guided hyperthermia: in vitro feasibility studies. Acad Radiol. 7, 2000, $1107-1115$.

[78] N. McDannold, S. L. Fossheim, H. Rasmussen, H. Martin, N. Vykhodtseva, K. Hynynen, Heat-ctivated lioposomal MR contrast agent: initial in vivo results in rabbit liver and kidney. Radiology. 230. 2004, 743 - 752.

[79] L. H. Lindner, H. M. Reinl, M. Schlemmer, R. Stahl, M. Peller, Paramagnetic thermosensitive liposomes for MRthermometry. Int J Hyperthermia. 21, 2005, $575-588$.

[80] S. Hey, M. De Smet, C. Stehning et al. Simultaneous T1 measurements and proton resonance frequency shift based thermometry using variable flip angles. Magn Reson Med. 67, 2012, 457 - 463.

[81] G. N. Chiu, S. A. Abraham, L. M. Ickenstein et al. Encapsulation of doxorubicin into thermosensitive liposomesvia complexation with the transition metal manganese. J Control Release. 104, 2005, 271288.

[82] C. Chen, D. Han, C. Cai, X. Tang, An overview of liposome lyophilization and its future potential. J Control Release, 142(3), 2010, 299-311, 2010.

[83] O. H. EL Naser, S. A. Yahiya, O. N. EL-Gazayerly, Effect of formulation design and freeze-drying on properties of fluconazole multilamellar liposomes. Saudi Pharm J. 18, 2010, 217-224.

[84] T. Lammers, W. E. Hennink, G. Storm, Tumour-targeted nanomedicines: principles and practice. Br J Cancer, 99(3), 2008, 392-397.

[85] S. Y. Wu, L. N. Putrl, M. Liang et al. Development of a novel method for formulating stable siRNA-loaded lipid particles for in vivo use. Pharm. Res. 26(3), 2009, 512-522. 